(ref. 2) we observe transfer from the $\mathrm{Er}^{+3}$ absorption bands near $490\left({ }^{4} \mathrm{~F}_{7 / 2}\right), 660\left({ }^{4} \mathrm{~F}_{9 / 2}\right), 810\left({ }^{4} \mathrm{I}_{9 / 2}\right)$, and $950 \mathrm{~m} \mu\left({ }^{4} \mathrm{I}_{11 / 2}\right)$; the term assignments follow those of $\mathrm{Er}^{+3}$ in $\mathrm{Y}_{2} \mathrm{O}_{3}$ (ref 4). The fluorescent intensity of $1 / 2 \% \mathrm{Tm}^{+3}$ in $\mathrm{Er}_{2} \mathrm{O}_{3}$ is more than 20

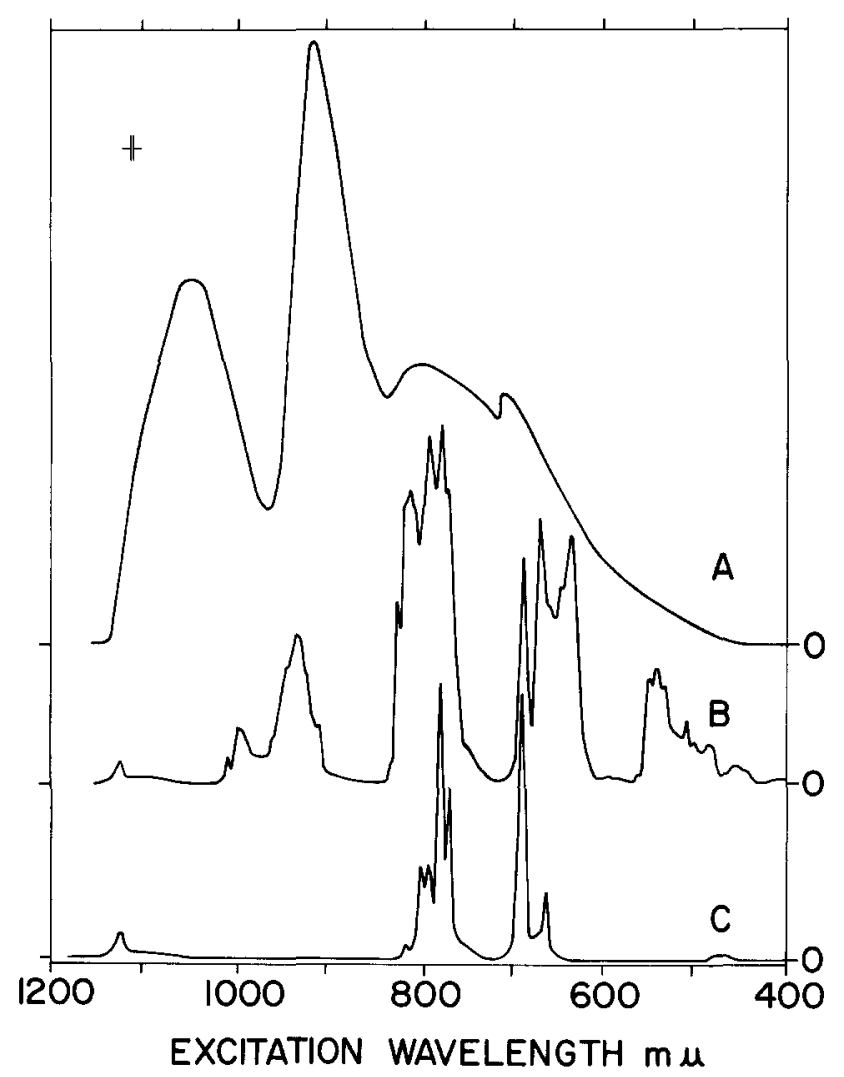

Fig. 2. $A$. Instrumental response. $B$. Excitation spectrum of the 1.93- $\mu$ fluorescence of 1/2-atom percent $\mathrm{Tm}^{+3}$ in $\mathrm{Er}_{2} \mathrm{O}_{3}$. $C$. Excitation spectrum of the $1.93-\mu$ fluorescence of $1 / 2$-atom percent $\mathrm{Tm}^{+3}$ in $\mathrm{Y}_{2} \mathrm{O}_{3}$. times as intense as the emission from $1 / 2 \% \mathrm{Tm}^{+3}$ in $\mathrm{Y}_{2} \mathrm{O}_{3}$ at $77^{\circ} \mathrm{K}$ under tungsten excitation.

The laser experiments were conducted with a sample approximately $1 \mathrm{~cm}$ in length with flat and parallel silvered ends using a fast gold-doped germanium detector. The threshold was less than 3 joules into a l-in. straight xenon flash lamp in close proximity to the liquid nitrogen dewar containing the sample. No laser operation could be obtained from the nearby fluorescent lines at an energy input of 20 joules.

The lifetime of the fluorescence is $2.9 \mathrm{msec}$ at $77^{\circ} \mathrm{K}$ with a build-up time of about $200 \mu \mathrm{sec}$. Nearthreshold laser emission is observed $50 \mu \mathrm{sec}$ after the $250-\mu \mathrm{sec}$ lamp pulse is over, an effect which is a consequence of this build-up time.

CW operation was observed using 500 -W quartziodine tungsten lamps as the pumping source. In the configuration used, the liquid nitrogen dewar containing the sample was surrounded by an array of 12 lamps. Threshold was reached with three lamps.

The crystals were grown by A. C. Pastor and K. Arita under the direction of R. C. Pastor. We thank D. Roberts for the use of the CW laser housing, C. R. Duncan for technical assistance, and J. Mikiska for the coatings.

\footnotetext{
${ }^{1}$ Supported by U.S. Air Force Electronic Technology Division, A.F. Avionics Laboratory Research and Technology Division, Wright-Patterson Air Force Base, Ohio, under Contract No. AF33(615)-1967.

${ }^{2}$ L. F. Johnson, L. G. Van Uitert, J. J. Rubin, and R. A. Thomas, Phys. Rev. 133, A494 (1964).

${ }^{3}$ J. B. Gruber, W. F. Krupke, and J. M. Poindexter, J. Chem. Phys. 41, 3363 (1964).

${ }^{4}$ P. Kisliuk, W. F. Krupke, and J. B. Gruber, J. Chem. Phys. 40, 3606 (1964).
}

\title{
LENSLESS FOURIER-TRANSFORM METHOD FOR OPTICAL HOLOGRAPHY
}

(x-ray diffraction microscopy; image synthesis;

"3-dimensional" photography; T/E)
George W. Stroke

The University of Michigan Ann Arbor, Michigan (Received 26 March 1965)
We have recently shown that a Fourier-transform hologram rather than a Gabor-Leith projection hologram was needed for attainment of high resolutions in wavefront-reconstruction imaging, in particular for microscopy applications at short, ultraviolet and $\mathrm{x}$-ray wavelengths. ${ }^{1,2,3}$ Heretofore in optics, Fourier-transformation required a focussing lens or mirror system: the transformation was carried out between the complex amplitude distribution of the field (say the $\overline{\mathbf{E}}$ field) in the pupil and the complex amplitude distribution of the field in the focal plane of the focussing system. Clearly the 
use of a Fourier-transforming lens-system defeats the advantages inherent in "lensless" holographic imaging. ${ }^{*}$

Here we show that the use of a point-reference coherent-background source in the principal plane of the object does indeed result in a Fourier-transform hologram, simply by projection onto a photographic plate. ${ }^{3}$ For shortness, we may call this arrangement a "lensless Fourier-transforming system", and the resulting hologram a "lensless Fourier-transform hologram".

The mathematical demonstration of the lensless Fourier-transforming property of the holographic system is straightforward. ${ }^{3}$ However, the physical principles can be readily demonstrated by discussion of Figs. 1 and 2. In Fig. 1 the lens $L$ produces a plane wavefront in the direction of the $z$ axis for the point-reference $R$, and an array of plane waves $\Sigma$ with different angles of incidence $\theta$ for the various points $P_{n}$ of the object. The resulting recording

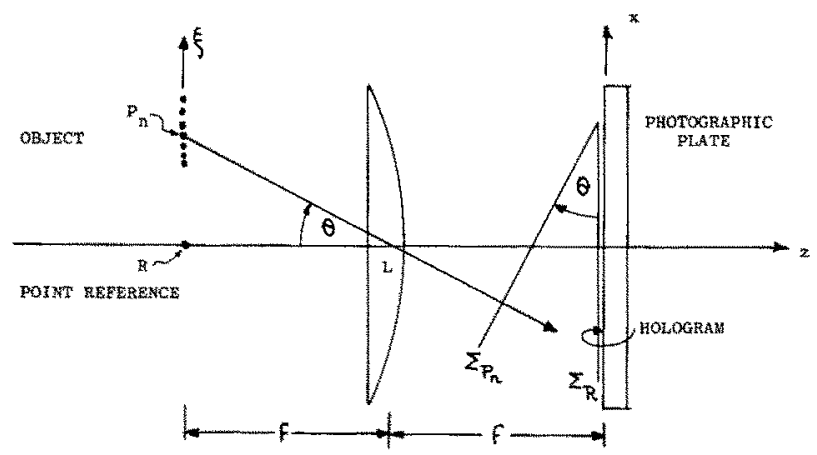

Fig. 1. Recording of a Fourier-transform hologram with a lens $L . \Sigma_{R}=$ reference wavefront.

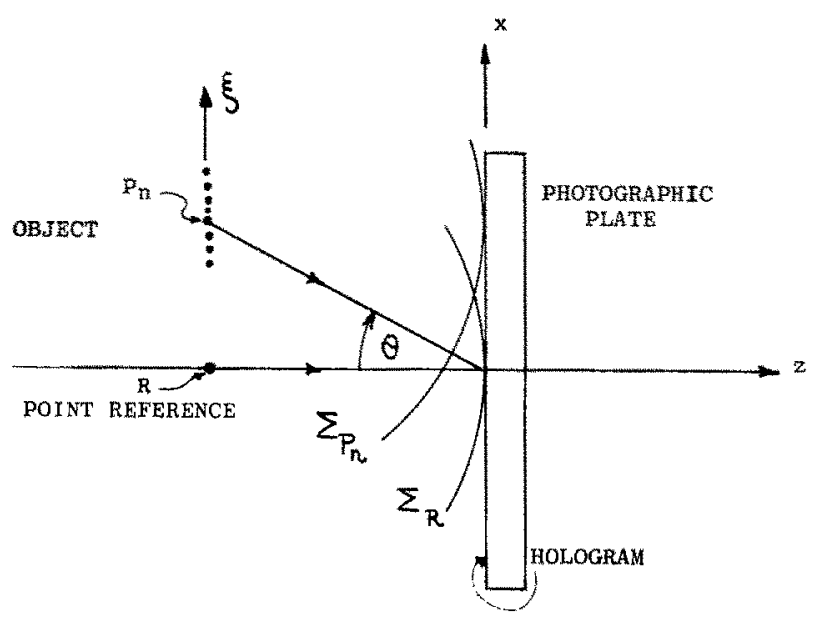

Fig. 2. Lensless recording of a Fourier-transform hologram. The two spherical wavefronts $\Sigma_{P_{n}}$ and $\Sigma_{k}$ have the same curvature. on the photographic plate is a Fourier-transform hologram. In Fig. 2 the hologram is obtained without a focussing element by interference of spherical waves $\Sigma$ of the same sphericity, rather than of plane waves as in Fig. 1. In the recording process, the spherical waves will cancel out by interference and the resulting hologram will in effect be identical to that of Fig. 1. Reconstruction of the image is simply obtained by Fourier-transformation of the hologram, ${ }^{1,3}$ for instance by placing the hologram into a collimated beam and recording the images in the focal plane of a lens. ${ }^{1,3}$

It is of particular interest to demonstrate that a lensless Fourier-transform hologram and a Fouriertransform reconstruction from it can indeed be obtained. Fig. 3 shows the reconstruction of a grating-like object from a hologram obtained with the lensless Fourier-transform hologram recording system of Fig. 2. Both the recording and the re-

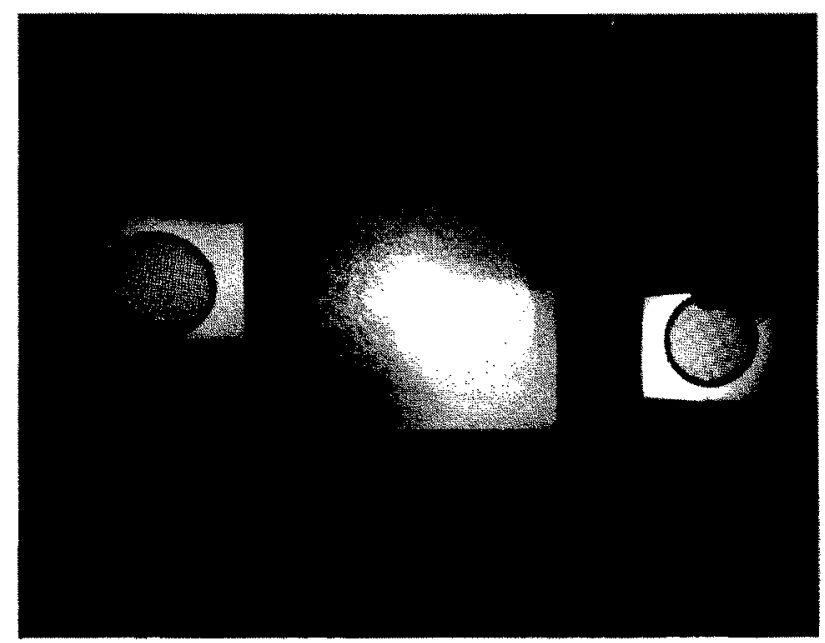

Fig. 3. Fourier-transform reconstruction of a $14 \mathrm{~mm}$ diam grid from a hologram recorded with the lensless arrangement shown in Fig. 2. The figure shows a single exposure of the two side-band images, as well as the unattenuated central image.

construction were carried out in 6328 - $\AA$ laser light. Both the resolution and the magnification of the lensless Fourier-transform systems are determined by the same considerations as in Fourier-transform systems using focussing elements.

We acknowledge the kind assistance of David G. Falconer with the theoretical aspects, that of $\mathrm{D}$. Brumm and A. Funkhouser with the experimental aspects of the work in our laboratory, and the generous support of the National Science Foundation for the pursuit of this work. 
${ }^{1}$ G. W. Stroke and D. G. Falconer, Phys, Letters 13, 306 (1964).

${ }^{2}$ G. W. Stroke and D. G. Falconer, Phys. Letters 15, No. $3(1$ April 1965).

${ }^{3}$ G. W. Stroke and D. G. Falconer, "Theoretical and Experi- mental Foundations of Wavefront-Reconstruction Imaging," in Sympasium on Optical and Electro-Optical Information Processing, ed. J. T. Tippett, L. C. Clapp, D. Berkowitz, and C. J. Koester (M.I.T. Press, 1964) in print.

${ }^{4}$ D. Gabor, Proc. Phys. Soc. (London) B64, 449 (1951).

\section{ZEEMAN EFFECT, FREQUENCY PULLING AND FREQUENCY PUSHING IN A SINGLE-MODE He-Ne LASER}

\author{
(axial magnetic field effect; gas pressure \\ $5 \mathrm{~mm} \mathrm{Hg}$; collision broadening of linewidth \\ parameter to $\sim 1200 \mathrm{Mc} / \mathrm{s} ; \mathrm{E}$ )
}

This Letter describes and interprets the influence of interferometer tuning on the frequency of the beat between the two Zeeman components in the radiation of an $\mathrm{He}-\mathrm{Ne}$ laser with applied axial magnetic field.

We have applied magnetic fields from 5 to $30 \mathrm{Oe}$, so that circularly polarized oscillations were observed (cf. ref. 1). The laser used was a dc-excited short planar He-Ne laser ${ }^{2}$ operating in a single axial mode $^{3}$ at $1.153-\mu$ wavelength. The total gas pressure was about $5-\mathrm{mm} \mathrm{Hg}$. The interferometer was thermally tuned; ${ }^{4}$ a thermocouple was used to measure the temperature of the wall of the tube.

The strong dependence of beat frequency on interferometer tuning is shown in Fig. 1, where the dc laser power output is also plotted. Because we used naturally occurring neon, the well-known single-mode tuning dip at line center ${ }^{5}$ is less pronounced in our plot. ${ }^{6.7}$ Only at interferometer resonances near threshold does the beat frequency appear to be proportional to the magnetic field strength. With a weak magnetic field and a high excitation level the beat signal at line center becomes distorted and finally disappears, as is indicated by the dotted part of the curve for $\eta_{1}$ and $1 A$ in Fig. 1 (cf. ref. 1).

The explanation of the beat frequency shift is based on the application of the theory of frequency "pulling" and frequency "pushing", as given by $\mathrm{Lamb}^{5}$ to the two Zeeman components separately. We have calculated $(\nu-\Omega) / \Delta \nu_{c}$ as a function of $(\Omega-\omega) / K u$, where $\nu$ is the oscillation frequency, $\Omega$ is the resonant frequency of the interferometer, $\Delta \nu_{c}$ is the interferometer width and $\omega$ is the peak frequency of the atomic resonance line, expressed as circular frequencies. ${ }^{5}$ The parameter $K u$ is pro-

\author{
P.T.Bolwijn \\ Physics Laboratory, State University Utrecht \\ The Netherlands \\ (Received 1 February 1965; in final form 26 April 1965)
}

portional to the Doppler width of the atomic line. A full Doppler width at half-maximum intensity of $800 \mathrm{Mc} / \mathrm{sec}$ has been assumed, and corresponds to a value of $K u \approx 3000 \mathrm{Mc} / \mathrm{sec}$. The theory also contains the parameter $\gamma_{a b}=\pi \Delta \nu_{n}$, where $\Delta \nu_{n}$ is the natural linewidth for the laser transition involved. The value of $\Delta \nu_{n}$ is not exactly known and may be several

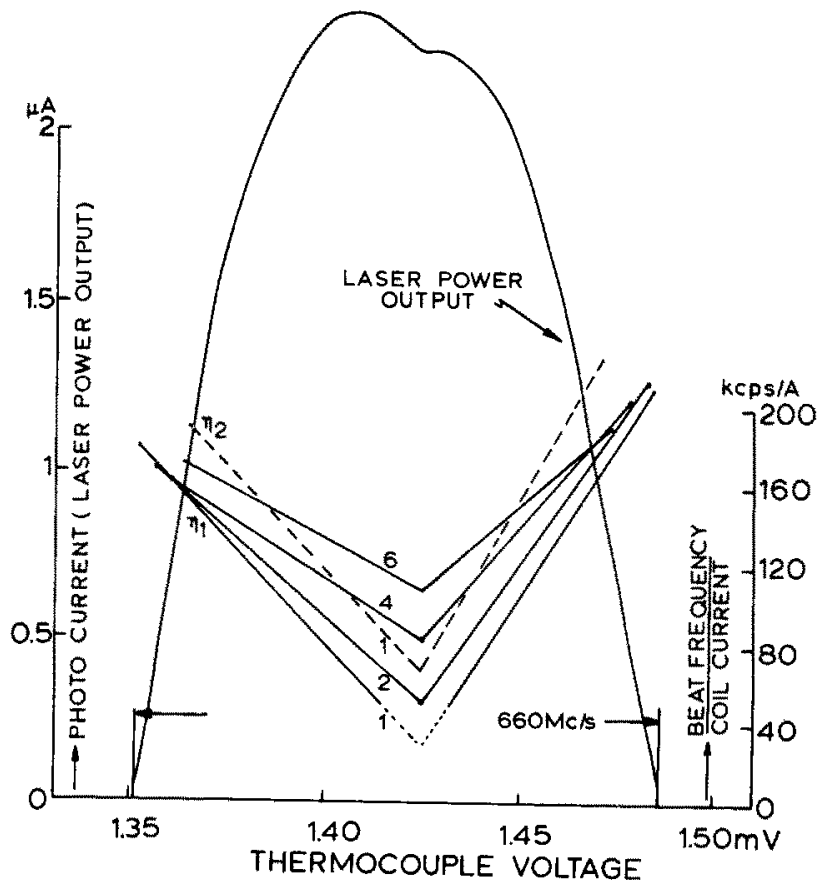

Fig. 1. The photocurrent and the ratio between Zeeman beat frequency and coil current vs thermocouple voltage. Coil currents used were $1,2,4$ and $6 \dot{A}$, giving magnetic fields of $5 \mathrm{Oe} / \AA$. The resonance frequency decreases with increasing thermocouple voltage. Relative excitation above threshold is characterized by parameter $\eta_{1}$ (drawn curves) and $\eta_{2}$ (dashed curve), with $\eta_{1}>\eta_{2}$. The laser power output is plotted for relative excitation $\eta_{1}$. 